Publisher: CSIRO; Journal: SH:Sexual Health

Article Type: research-article; Volume: 7; Issue: 3; Article ID: SH10068

DOI: 10.1071/SH10068; TOC Head:

\title{
A trial of the distribution of chlamydia self-collection postal specimen kits from Australian community pharmacies
}

\author{
Lynne Emmerton $^{\mathrm{A}, \mathrm{E}}$, Monika Buhrer Skinner ${ }^{\mathrm{B}, \mathrm{C}}$, Elliroma Gardiner $^{\mathrm{A}}$, Lisa Nissen ${ }^{\mathrm{A}}$ and Joseph \\ Debattista $^{\mathrm{D}}$

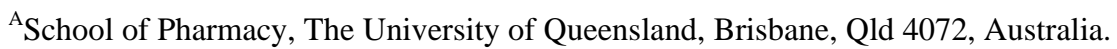

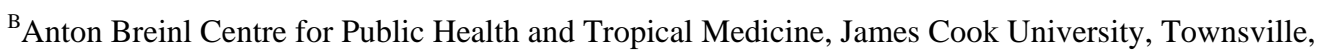 \\ Qld, Australia.
}

${ }^{\mathrm{C}}$ Institute of Primary Health and Ambulatory Care, Townsville Health Service District, Queensland Health, Townsville, Qld, Australia.

${ }^{\text {D}}$ Metro North Health and Sunshine Coast Health Service District, Queensland Health, Brisbane, Qld, Australia.

${ }^{\mathrm{E}}$ Corresponding author. Email: l.emmerton@pharmacy.uq.edu.au

\section{SH10068}

L. Emmerton et al.

Running head title?

The involvement of pharmacies in offering non-invasive sexually transmissible infection (STI) testing is considered important given their presence in the community, long opening hours, and credibility as health-care providers and promoters. In the UK, pharmacy-based distribution of chlamydia selfcollection specimen kits was successfully trialled, in over 200 Boots pharmacies in London in late 2005. ${ }^{1,2}$ Clients aged 16 years and over in the UK may now purchase the specimen kits and mail their urine sample to the laboratory for testing. ${ }^{3}$

We report some outcomes of the pharmacy sub-study of a Queensland Chlamydia Testing Program that explored various modes of distribution for specimen self-collection kits. Availability of specimen collection kits from pharmacies may enhance the uptake of testing services. In contrast to other countries, however, Australian postal regulations do not permit mailing of liquid specimens. Development of the regulation-compliant kit used in this study has been reported elsewhere. ${ }^{4,5}$ Briefly, a transport medium for urine samples, a self-collection postal kit, as well as a clinical management system, including notification of the test result by telephone or text message and referral of positive cases for treatment, were developed and tested for functionality.

The community pharmacy arm of this study investigated (1) the feasibility of community pharmacies for distribution of specimen kits and (2) associations between risk-based screening and test results. Four Queensland community pharmacies participated, selected to target sectors of the community lacking opportunity for chlamydia testing. Each pharmacy was issued 75 specimen 
Publisher: CSIRO; Journal: SH:Sexual Health

Article Type: research-article; Volume: 7; Issue: 3; Article ID: SH10068

DOI: 10.1071/SH10068; TOC Head:

collection kits (Fig. 1), each with instructions for collection and mailing of the specimen and a codematched questionnaire exploring published risk factors. In accordance with the ethical approval for this study, pharmacy staff were trained to offer kits to clients 16 years or older, fluent in English and presenting for a sexual health-related product or consultation. The accompanying questionnaires were completed by clients in the pharmacy, submitted in a sealed envelope and retrieved by the researchers. Code-matched chlamydia test results from the testing centre were compared with the nominallyscored questionnaire data. Specimens were tested with the polymerase chain reaction assay, and results reported back to clients in the manner requested when the specimens were mailed in.

As part of the evaluation, pharmacy staff members were interviewed in person (by telephone for a remote pharmacy) at the conclusion of the study regarding their experiences with the processes.

Kits were distributed over a 4-month period in 2008. Of 300 kits, 156 were distributed to clients (1-75 per pharmacy), with 18 persons submitting specimens for testing (12\%). This return rate was comparable to the average across the eight arms of the parent study ${ }^{5}$. Four of the 18 specimens received were reactive for Chlamydia trachomatis. Forty-four risk-assessment questionnaires were retrieved (28\% of the distributed kits), indicating that these clients averaged 25 years (range 16-48 years) and were predominantly females $(n=41)$. Sixteen respondents $(37 \%)$ were identified by their questionnaire scores as 'at-risk' of testing positive for chlamydia. Comparing the test results with the questionnaire risk scores, higher risk scores were noted for two of the four positive cases.

Risk behaviours reported in the questionnaires included multiple partners in the past year and symptoms suggestive of an STI. Condom use was sporadic. Twenty-five of the 44 participants reported having been previously tested for chlamydia; of these, six recalled a previous positive test and seven were unsure.

Interviews with all available pharmacy staff identified support for the study, but there were commitment issues relating to workload and, in some cases, no staff member taking responsibility for 'driving' the distribution of the specimen collection kits. Another factor limitation was the restriction of advertising to in-store posters and leaflets. This required pharmacy staff to verbally introduce the concept to potential participants. Despite the training and privacy measures incorporated into the study design, some staff members reported a level of discomfort in this process.

The distribution of the postage-compliant specimen kits from our sample of community pharmacies was considered moderately successful in facilitating access to this testing service by an at-risk sector of the population, as determined by the proportion of kits issued and the proportion of specimens submitted. However, pharmacy participation was highly variable, and we recommend staff education to improve motivation to promote kits and approval for public advertising of the service. The association between the risk-assessment questionnaires and test results was inconclusive, due to the 
Publisher: CSIRO; Journal: SH:Sexual Health

Article Type: research-article; Volume: 7; Issue: 3; Article ID: SH10068

DOI: 10.1071/SH10068; TOC Head:

small sample size. We suggest that the risk criteria may be useful in other ways, such as public awareness campaigns, to encourage self-identification for testing.

\section{Conflicts of interest}

xxx.

\section{Acknowledgements}

We acknowledge Ms Rose Gordon for technical assistance. This project was funded by the Australian

Commonwealth Government, as part of a National Chlamydia Pilot Program testing the effectiveness of several models for chlamydia testing in Australia. This project will assist in developing possible recommendations for a National Chlamydia Program.

\section{References}

<jrn>1 Anonymous. Minister launches Boots Chlamydia service. Pharm J 2005; 275: 596.</jrn>

$<$ eref $>2$ UK Department of Health. First month of Chlamydia screening on the High Street a success, UK.

Medical News Today 2006; Jan 3. Available online at:

http://www.medicalnewstoday.com/medicalnews.php?newsid=35633 [verified May 23, 2010].</eref>

$<$ eref $>3$ Boots Pharmacy Chlamydia Screening Service. Chlamydia. Available online at:

http://www.boots.com/en/Clamelle-Chlamydia-Test-Kit_852398/[verified May 23, 2010].</eref>

<jrn>4 Bialasiewicz S, Whiley DM, Buhrer-Skinner M, Muller R, Debattista J, Nissen MD, et al. A novel gelbased method for self-collection and ambient temperature postal transport of urine for PCR detection of Chlamydia trachomatis. Sex Transm Infect 2009; 85: 102-5. doi:10.1136/sti.2008.032607</jrn>

$<$ jrn>5 Buhrer-Skinner M, Muller R, Bialasiewicz S. The check is in the mail: piloting a novel approach to Chlamydia trachomatis testing using self-collected, mailed specimen. Sex Health 2009; 6: 163-9. doi:10.1071/SH08076 < $<$ jrn>

Manuscript received 9 June 2010, accepted 10 June 2010

Fig. 1. Study method. CPHN, xxx; UQ, University of Queensland. 